\title{
Random Strain Fluctuations as Dominant Disorder Source for High-Quality On-Substrate Graphene Devices
}

\author{
Nuno J. G. Couto, ${ }^{1}$ Davide Costanzo, ${ }^{1}$ Stephan Engels, ${ }^{2}$ Dong-Keun Ki, ${ }^{1}$ Kenji Watanabe ${ }^{3}$ \\ Takashi Taniguchi, ${ }^{3}$ Christoph Stampfer, ${ }^{2}$ Francisco Guinea, ${ }^{4}$ and Alberto F. Morpurgo ${ }^{1, *}$ \\ ${ }^{1}$ Département de Physique de la Matière Condensée (DPMC) and Group of Applied Physics (GAP), \\ University of Geneva, 24 Quai Ernest-Ansermet 1211 Genève 4, Switzerland \\ ${ }^{2}$ JARA-FIT and 2nd Institute of Physics, RWTH Aachen University, 52074 Aachen, Germany \\ and Peter Grünberg Institute (PGI-9), Forschungszentrum Jülich, 52425 Jülich, Germany \\ ${ }^{3}$ National Institute for Materials Science, 1-1 Namiki, Tsukuba 305-0044, Japan \\ ${ }^{5}$ Instituto de Ciencia de Materiales de Madrid, CSIC, Cantoblanco, E-28015 Madrid, Spain \\ (Received 2 July 2014; revised manuscript received 27 August 2014; published 30 October 2014)
}

We perform systematic investigations of transport through graphene on hexagonal boron nitride (hBN) substrates, together with confocal Raman measurements and a targeted theoretical analysis, to identify the dominant source of disorder in this system. Low-temperature transport measurements on many devices reveal a clear correlation between the carrier mobility $\mu$ and the width $n^{*}$ of the resistance peak around charge neutrality, demonstrating that charge scattering and density inhomogeneities originate from the same microscopic mechanism. The study of weak localization unambiguously shows that this mechanism is associated with a long-ranged disorder potential and provides clear indications that random pseudomagnetic fields due to strain are the dominant scattering source. Spatially resolved Raman spectroscopy measurements confirm the role of local strain fluctuations, since the linewidth of the Raman 2D peak-containing information of local strain fluctuations present in graphene-correlates with the value of maximum observed mobility. The importance of strain is corroborated by a theoretical analysis of the relation between $\mu$ and $n^{*}$ that shows how local strain fluctuations reproduce the experimental data a quantitative level, with $n^{*}$ being determined by the scalar deformation potential and $\mu$ by the random pseudomagnetic field (consistently with the conclusion drawn from the analysis of weak localization). Throughout our study, we compare the behavior of devices on $\mathrm{hBN}$ substrates to that of devices on $\mathrm{SiO}_{2}$ and $\mathrm{SrTiO}_{3}$, and find that all conclusions drawn for the case of $\mathrm{hBN}$ are compatible with the observations made on these other materials. These observations suggest that random strain fluctuations are the dominant source of disorder for high-quality graphene on many different substrates, and not only on hexagonal boron nitride.

DOI: 10.1103/PhysRevX.4.041019

\section{INTRODUCTION}

Hexagonal boron nitride $(\mathrm{hBN})$ substrates enable the fabrication of graphene devices [1-4], exhibiting extremely high carrier mobility values, and leading to the observation of new, interesting physical phenomena [5-9]. The precise microscopic reason for the quality of these devices, however, has not yet been established, nor is there an understanding of what the dominant microscopic physical mechanism responsible for the remnant disorder is. Here, we perform a systematic study of a large number of such

\footnotetext{
* Corresponding author. Alberto.Morpurgo@unige.ch

Published by the American Physical Society under the terms of the Creative Commons Attribution 3.0 License. Further distribution of this work must maintain attribution to the author(s) and the published article's title, journal citation, and DOI.
}

Subject Areas: Condensed Matter Physics, Graphene, Mesoscopics devices and provide considerable evidence - both experimentally and theoretically - that random local strain fluctuations in the graphene lattice are the dominant microscopic source of disorder.

Many different techniques are currently used for the production of graphene devices, and the dominant source of disorder depends on the specific type of device considered. We confine our attention to high-quality devices, based on graphene monolayers exfoliated from natural graphite and transferred to be in direct contact with a substrate material, not exposed to damaging agents (such as electron or ion beams, ultraviolet radiation, or aggressive chemical environments). Even so, many different physical mechanismssuch as charged impurities at the substrate surface, adsorbates acting as resonant scatterers, structural defects such as vacancies, strain fluctuations, and more-have been considered as possible sources of disorder [10]. Conducting targeted experiments to identify the dominant source in any 
given individual device is virtually impossible, and information can only be extracted by analyzing the statistical behavior of many devices realized under controlled conditions. Experiments have been performed to intentionally introduce one specific type of disorder in graphene (e.g., charged impurities, by depositing an increasingly large number of potassium atoms on a graphene layer [11], or vacancies, by bombarding graphene with an increasingly large dose of heavy ions [12]) while monitoring the resulting variations in the electronic properties. This work is useful to test specific predictions of theories describing disorder of different natures, but does not enable the determination of the physical mechanism causing the disorder initially present in the devices. Considerable research has been devoted to analyze the dependence of the conductivity of graphene $(\sigma)$ on carrier density $(n)$, without, however, solving the existing controversies, mainly because the measured $\sigma(n)$ curves are consistent with the functional dependence predicted by models describing different sources of disorder. Despite the work of many different research groups, there is not even an established consensus for the most common devices on $\mathrm{SiO}_{2}$, as to whether the dominant disorder potential is short or long ranged (i.e., whether it has a range comparable to the lattice spacing or much longer) [13-20].

Our work exploits a combination of different experimental techniques, together with the statistical analysis of a large number of devices on hBN substrates, looking at both the carrier mobility $\mu$ and the width of the resistance peak around charge neutrality $n^{*}$. While the best graphene-on-hBN devices exhibit impressively high mobility values, more modest values are also commonly found, so that the resulting broad range of electrical characteristics allows the identification of correlations between different quantities. We find an unambiguous correlation between the carrier mobility $\mu$ and the width of the resistance peak around charge neutrality $n^{*}$-with $\mu \propto\left(n^{*}\right)^{-1}$ - extending over nearly 2 orders of magnitude, which demonstrates that the physical mechanism limiting the mobility is the same one causing charge inhomogeneity. To identify this mechanism, we perform weak-localization measurements to extract several characteristic scattering times, such as the intervalley scattering time $\tau_{i v}$ and the time $\tau_{*}$ associated with the breaking of the effective, single-valley time-reversal symmetry. For all charge carrier densities, $\tau_{i v}$ is much longer than $\tau$, the elastic scattering time extracted from the carrier mobility. This finding directly establishes that the mobility is limited by intravalley scattering caused by long-ranged potentials, confining the possible microscopic mechanisms to charged impurities and random strain fluctuations in the graphene lattice.

Two independent observations indicate that local strain fluctuations dominate. First, weak-localization measurements show that $\tau_{*}$ and $\tau$ nearly coincide, a finding that is readily explained if pseudomagnetic fields due to local strain are the dominant source of elastic scattering, but that cannot be explained by the charged impurity mechanism. Second, we directly probe local strain fluctuations with confocal Raman experiments [21] and show experimentally that larger strain fluctuations limit the maximum mobility that can be observed in transport measurements. Based on this evidence, we analyze theoretically the linear relation between $1 / \mu$ and $n^{*}$-which was previously observed in devices exposed to potassium atoms and taken to be an indication of charge impurity scattering - and show that such a relation can be explained quantitatively invoking random strain fluctuations only. According to this same analysis, it is the random pseudomagnetic field originating from strain fluctuations, and not the deformation potential, that gives the dominant contribution to the scattering of charge carriers, in agreement with the conclusion drawn from the analysis of weak localization. Whereas most of our work has focused on graphene-on-hBN devices, we have also looked at devices on $\mathrm{SiO}_{2}$ and $\mathrm{SrTiO}_{3}$ substrates and found that the observations made on these devices are fully compatible with the conclusions drawn for hBN, which points to the relevance of random strain fluctuations under rather broad experimental conditions for high-quality graphene devices on different substrates.

\section{EXTRACTING $\boldsymbol{\mu}$ AND $\boldsymbol{n}^{*}$ FOR GRAPHENE DEVICES ON hBN}

The fabrication of graphene-on-hBN devices relies on a technique described in the literature [1]. We exfoliate hBN crystals onto a heavily doped, oxidized $\mathrm{Si}$ wafer. Graphene flakes extracted from natural graphite are transferred onto a hBN crystal, following the procedure of Ref. [1]. Metallic contacts $(\mathrm{Ti} / \mathrm{Au}, 10 / 75 \mathrm{~nm})$ are defined by electron-beam lithography, evaporation, and lift-off [see Fig. 1(a)]. We find that "bubbles" and "folds" form when transferring graphene on $\mathrm{hBN}$ (as in Refs. [2,3,22]) : achieving high $\mu$ requires etching Hall bar devices in parts of the flakes where no such defects are present (regions with bubbles exhibit lower $\mu$, comparable to $\mathrm{SiO}_{2}$ devices). After an electrical characterization at $4 \mathrm{~K}$, we perform different low-temperature thermal annealing steps (at up to $150{ }^{\circ} \mathrm{C}-250^{\circ} \mathrm{C}$, in an environment of $\mathrm{H}_{2} / \mathrm{Ar}$ at $100 / 200 \mathrm{sccm}$ ) and check the low-temperature transport characteristics each time. We find that the initial annealing step always results in a mobility increase (a factor of 2 in the very best cases), whereas subsequent annealing leads to a decrease in $\mu$, eventually to values similar to those obtained on $\mathrm{SiO}_{2}$ [23].

We analyze approximately 15 distinct Hall-bar devices. Mobility values (at $4.2 \mathrm{~K}$ ) between 30.000 and $80.000 \mathrm{~cm}^{2} / \mathrm{V} \mathrm{s}$ at a carrier density of a few $10^{11} \mathrm{~cm}^{-2}$ are regularly found. Integer quantum Hall plateaus with $\sigma_{\text {Hall }}=4(1 / 2+N) e^{2} / h(N$ integer $)$ are fully developed starting from $B=1 \mathrm{~T}$, and broken symmetry quantum Hall states with Hall conductivity $\sigma_{\text {Hall }}= \pm 1 e^{2} / h$ appear from 

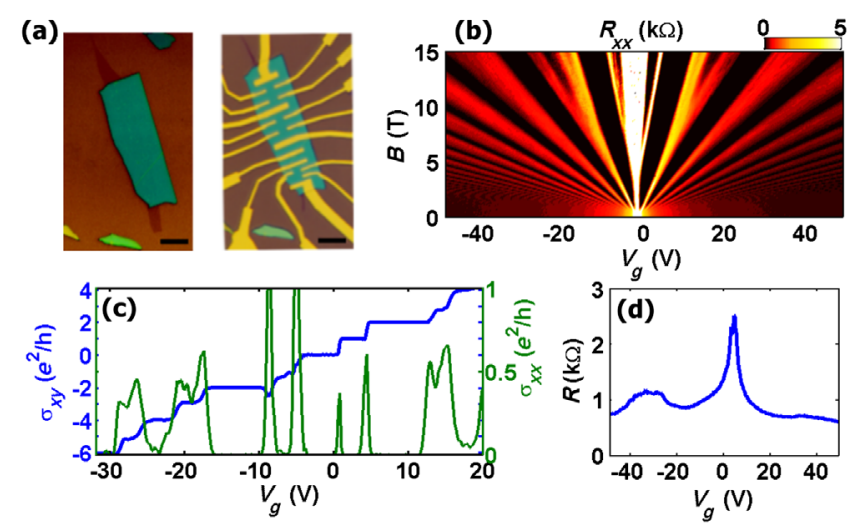

FIG. 1. (a) Optical microscope image of a monolayer graphene flake on top of a 30-nm-thick hBN crystal before (left) and after (right) depositing metal contacts (the scale bars are $5 \mu \mathrm{m}$ ). (b) Longitudinal resistance $R_{x x}$ as a function of $V_{g}$ and $B$ showing quantum Hall states originating from the lifting of the single-particle degeneracy already at $B \simeq 8 \mathrm{~T}$. (c) Hall (blue line) and longitudinal (green line) conductivity as a function of $V_{g}$ measured at $B=15 \mathrm{~T}$, showing full degeneracy lifting of Landau level $N=0,1$. (d) Resistance of a graphene device whose edge was aligned to that of the hBN substrate, showing the emergence of satellite Dirac peaks (well developed for negative $V_{g}$ and less pronounced for positive $V_{g}$ ). All measurements have been taken at $T=250 \mathrm{mK}$.

$B=8 \mathrm{~T}$ [see Fig. 1(b)]. Full degeneracy lifting of the $N=0$ and $N= \pm 1$ Landau levels is observed below $15 \mathrm{~T}$ [Fig. 1(c)]. In devices where the lattices of graphene and hBN are intentionally aligned, we observe the effect of a superlattice potential, with the appearance of satellite Dirac peaks in the measured $R\left(V_{g}\right)$ curve [Fig. 1(d)] [7-9]. These results indicate that our devices have quality comparable to those fabricated using a similar procedure, reported in the literature.

To evaluate the quality of our graphene-on-hBN devices, we focus on the low- $T$ mobility $\mu$ and on the width $n^{*}$ of the minimum in the conductivity. The mobility $\mu$ measures the elastic scattering time $\tau$ responsible for momentum relaxation, whereas $n^{*}$ quantifies the potential fluctuations experienced by electrons in graphene [10,24]. Since these potential fluctuations are not $a$ priori the dominant source of elastic scattering, there is no reason to assume that $\mu$ and $n^{*}$ are related. Experimentally, the carrier mobility is obtained from $\mu=\sigma / n e$ [see Fig. 2(a)], with the density of charge carriers $n$ obtained through the Hall resistance. To extract $n^{*}$, we plot $\log (\sigma)$ as a function of $\log (n)$ and determine at which $n$ the constant value of $\log (\sigma)$ measured at low density crosses the value of $\log (\sigma)$ extrapolated (linearly) from high density [as shown in Fig. 2(b)]. The mobility is estimated for $n>n^{*}$.

Figure 2(c) shows $\mu$ as a function of $n^{*}$ for all devices, measured either immediately after fabrication or after a subsequent annealing step. The presence of a correlation between $\mu$ and $n^{*}$ is unambiguous: devices with smaller
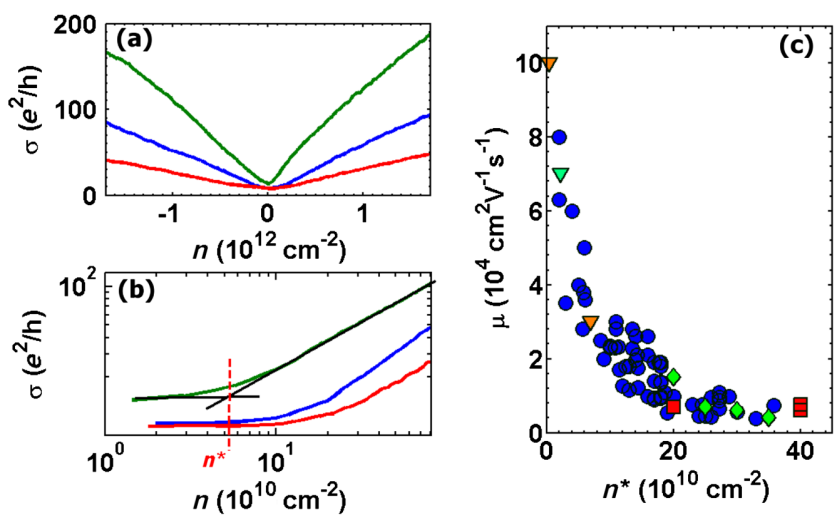

FIG. 2. Conductivity $\sigma$ of a graphene monolayer on $\mathrm{hBN}$ as a function of carrier density $n$ in linear (a) and double-logarithmic (b) scale, measured after fabrication (blue line), after a first annealing at $150{ }^{\circ} \mathrm{C}$ (green line), and after a second annealing at $250^{\circ} \mathrm{C}$ (red line). Panel (b) also illustrates the procedure to extract the value of $n^{*}$. (c) The blue full circles represent the lowtemperature mobility $\mu$ (plotted versus $n^{*}$ ) for all the 15 grapheneon-hBN devices realized in our laboratory, measured after fabrication or after annealing. The triangles represent data for graphene on hBN extracted from Refs. [1,5] (orange triangles) and from Ref. [2] (green triangle). The green diamonds and red squares are from devices realized in our laboratory on $\mathrm{SiO}_{2}$ and $\mathrm{SrTiO}_{3}$ substrates, respectively.

density fluctuations have larger mobility. For hBN devices fabricated in our laboratory, this correlation extends from $\mu$ values of $5.000 \mathrm{~cm}^{2} / \mathrm{V} \mathrm{s}$ (for devices after multiple annealing steps, see below) to $80.000 \mathrm{~cm}^{2} / \mathrm{V} \mathrm{s}$. Results reported in the literature $[1,2,5]$ quantitatively fit the same trend, extending the range to $\mu=100.000 \mathrm{~cm}^{2} / \mathrm{V} \mathrm{s}$. Plotting $1 / \mu$ versus $n^{*}$ [Fig. 3(a)] shows that the relation between these two quantities is essentially linear. To reduce the statistical fluctuations, we subdivide the $n^{*}$ axis into eight different intervals and plot the inverse averaged mobility as a function of the average charge density fluctuations [Fig. 3(b)], which makes the linear scaling of $1 / \mu$ with $n^{*}$ apparent.

We emphasize that neither the occurrence of the relation between $1 / \mu$ and $n^{*}$ nor its approximate linearity is obvious a priori. Indeed, it has been shown that when intentionally creating carbon vacancies, no such relation is observed, because in that case vacancies are the dominant mechanism responsible for the suppression of the carrier mobility, but they are not the dominant mechanism causing charge inhomogeneity [12]. Our observations, therefore, unambiguously establish that scattering of charge carriers and charge inhomogeneity in devices on $\mathrm{hBN}$ are caused by the same microscopic mechanism. Additionally, the linearity of the $1 / \mu-n^{*}$ relation is not trivial: we measure several graphene bilayer devices on $\mathrm{hBN}$ and $\mathrm{SiO}_{2}$ and find that a relation between $1 / \mu$ and $n^{*}$ also occurs in that case, but the relation is quadratic and not linear (see Appendix B). These considerations make clear that a quantitative analysis 

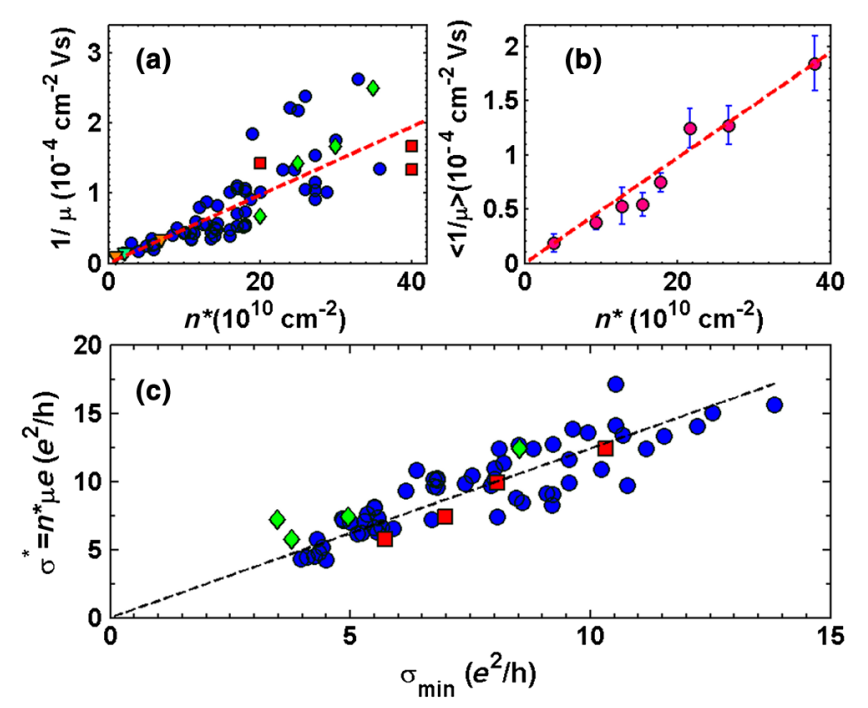

FIG. 3. (a) Same data as those of Fig. 2(c) plotted as $1 / \mu-n^{*}$, showing an overall linear relation. (b) Average inverse mobility as function of $n^{*}$ (obtained as indicated in the main text), showing clearly the linearity of the relation. In (a) and (b) the dashed lines are a linear fit to the data, $(1 / \mu)=(h / e) n^{*} \times 0.118$. (c) minimum conductivity $\sigma^{*}=n^{*} e \mu$, calculated from the estimated carrier density fluctuations $n^{*}$ and mobility $\mu$, and plotted as a function of measured minimum conductivity. The excellent overall agreement (the dashed line has slope 1) confirms the correctness of the procedures used to extract $n^{*}$ and $\mu$ from the measurements.

of the $1 / \mu-n^{*}$ relation can provide important information. Note that a correlation similar to the one shown in Figs. 3(a) and 3(b) has been reported for graphene covered by ionized potassium atoms, which do generate disorder consistent with the charged impurities mechanism [11]. On this basis, one may be tempted to conclude that charged impurities are also the dominant source of disorder for graphene on hBN. As we show below, however, the $1 / \mu-n^{*}$ correlation is also qualitatively and quantitatively compatible with the effect of random strain fluctuations in graphene, and discriminating between charged impurities and strain is the main goal of the remainder of this paper. Before coming to that, we notice that, rather surprisingly, the $1 / \mu-n^{*}$ correlation is also fulfilled by devices on different substrate materials, whose data points - the red and green dots in Fig. 2(c) represent data obtained from graphene on $\mathrm{SiO}_{2}$ and $\mathrm{SrTiO}_{3}$ [19] — fall on the curve defined by the results obtained for graphene devices on hBN [25].

\section{CHARACTERISTIC SCATTERING TIMES REVEAL THE ORIGIN OF DISORDER}

Having established that scattering of charge carriers and carrier density inhomogeneities are caused by the same microscopic mechanism, we can gain additional insight by analyzing weak localization to extract all the relevant scattering times for graphene on hBN [26-28]. Our first goal is to compare the intervalley scattering time $\tau_{i v}$ to the elastic scattering time $\tau$ determined from the carrier mobility. Either $\tau_{i v} \simeq \tau$, implying that the mobility is determined by intervalley scattering processes (i.e., the dominant source of disorder is short-range potentials), or $\tau_{i v} \gg \tau$, indicating that $\mu$ is limited by intravalley scattering (i.e., long-range disorder potentials dominate). Surprisingly, this straightforward argument has not been used systematically in previous work to identify the dominant disorder, nor has it been suggested in theoretical work (for an exception, see Ref. [29] dealing with rather low mobility devices, $\mu \simeq 1.000 \mathrm{~cm}^{2} / \mathrm{V} \mathrm{s}$ ).

Figure 4(a) shows the low-field magnetoresistance of a Hall bar device with $\mu \simeq 60.000 \mathrm{~cm}^{2} / \mathrm{V} \mathrm{s}$, for different values of $V_{g}$ around $V_{g}=8 \mathrm{~V}$, at $T=250 \mathrm{mK}$. A narrow dip in conductivity (width $\simeq 1 \mathrm{mT}$ or less) is seen around $B=0 \mathrm{~T}$, originating from weak localization. Aperiodic conductance fluctuations due to random interference are also visible, which we suppress by averaging measurements taken for slightly different $V_{g}$ values [30]. "Ensemble-averaged" curves obtained in this way around three different $V_{g}$ values are shown in Fig. 4(b). We perform similar measurements at several different temperatures and analyze the ensemble-averaged low-field magnetotransport up to $T=10 \mathrm{~K}$.

To analyze the data, we follow the same procedure used in previous studies of the quantum correction to the conductivity done on graphene on $\mathrm{SiO}_{2}$ substrates [28,29] and on epitaxial graphene on $\mathrm{SiC}$ [31]. Specifically, the data are fit to existing theory [26], from which we extract the intervalley scattering time $\tau_{i v}$, the phase coherence time $\tau_{\phi}$, and the time $\tau_{*}$ needed to break
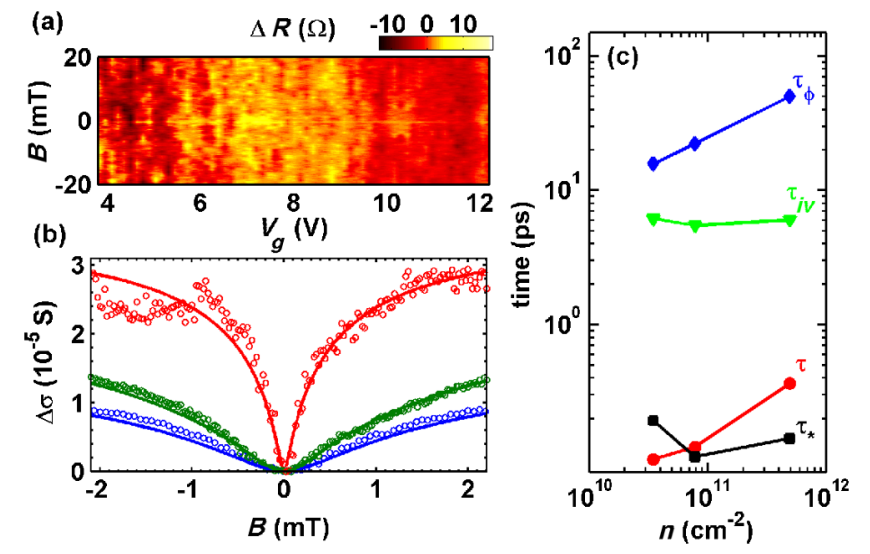

FIG. 4. (a) $B$ and $V_{g}$ dependence of the resistivity measured at $T=250 \mathrm{mK}$. (b) The circles represent magnetoconductivity curves $\Delta \sigma(B)$ that have been ensemble averaged, by averaging traces in a range of gate voltages around $V_{g}=-7$ (blue circles), 7 (green circles), and $30 \mathrm{~V}$ (red circles), to suppress samplespecific fluctuations. The continuous lines are fit to the theory of weak localization in graphene. (c) Characteristic times extracted at $250 \mathrm{mK}$ for different values of carrier density, either from the fit of weak-localization curves $\left(\tau_{\phi}, \tau_{i v}\right.$, and $\left.\tau_{*}\right)$ or from the conductivity $(\tau)$. The elastic scattering time $\tau$ is always at least 1 order of magnitude smaller than the intervalley scattering time $\tau_{i v}$. 
effective single-valley time-reversal symmetry [27], using the equation

$$
\begin{aligned}
\Delta \sigma(B)= & \frac{e^{2}}{\pi h}\left[F\left(\frac{\tau_{B}^{-1}}{\tau_{\phi}^{-1}}\right)\right. \\
& \left.-F\left(\frac{\tau_{B}^{-1}}{\tau_{\phi}^{-1}+2 \tau_{i v}^{-1}}\right)-2 F\left(\frac{\tau_{B}^{-1}}{\tau_{\phi}^{-1}+\tau_{i v}^{-1}+\tau_{*}^{-1}}\right)\right] .
\end{aligned}
$$

Here, $F(z)=\ln z+\psi\left(0.5+z^{-1}\right), \psi(x)$ is the digamma function, $\tau_{B}^{-1}=4 e D B / \hbar$, and $D=v_{F}^{2} \tau / 2$. In fitting the magnetotransport curves at different temperatures, we allow $\tau_{\phi}$ to vary-since the phase coherence time does increase with lowering $T$-and we constrain the other scattering times to be constant in the range investigated $(250 \mathrm{mK}$ and $10 \mathrm{~K})$. We obtain satisfactory agreement in all cases with a single set of values for $\tau_{i v}$ and $\tau_{*}$ (the elastic scattering time $\tau$-also constant as a function of $T$-is obtained from the measurements of the conductivity, and is not a fitting parameter).

Figure 4(c) shows the hierarchy of the relevant times at $T=250 \mathrm{mK}$, the lowest temperature reached in the experiments, for three different values of $n$. At this temperature, $\tau_{\phi}$ is much larger than $\tau_{i v}$, which is why weak localization is observed ( $\tau_{\phi}$ eventually becomes shorter than $\tau_{i v}$ as $T$ reaches $10 \mathrm{~K}$, so that weak antilocalization becomes visible, in conformity with theoretical expectations, and as found previously for graphene on $\mathrm{SiO}_{2}[28,29]$ ). More importantly, throughout the density range investigated, $\tau_{i v} \gg \tau$ by at least 1 order of magnitude (and by nearly 2 orders of magnitude at low $n$ ). This last observation implies that intravalley scattering is the process limiting $\mu$, a result that - in conjunction with previous measurements on graphene on $\mathrm{SiO}_{2}[28,29]$ — holds at least in the mobility range between 1.000 and $80.000 \mathrm{~cm}^{2} / \mathrm{V} \mathrm{s}$. We conclude that weak-localization measurements unambiguously show that the dominant source of disorder for exfoliated monolayer graphene on $\mathrm{hBN}$ (and $\mathrm{SiO}_{2}$ ) substrates is associated with long-ranged potentials (motivated by this conclusion, we have also recently studied weak localization on highquality graphene bilayer devices on hBN substrates, and in that case as well we have unambiguously come to the same conclusion, namely, that it is intravalley scattering processes that are limiting the carrier mobility [32]).

The results of the weak-localization measurements also provide a clear indication as to which of the two sources of long-range disorder (charged impurities at the substrate surface $[13,33,34]$ and random strain fluctuations in the graphene lattice [35]) plays the most relevant role. Specifically, the analysis of weak localization shows that $\tau \simeq \tau_{*}$ within a factor of 2-3, for all carrier density ranges investigated [Fig. 4(c)], a finding that is naturally explained by strain. Indeed, strain generates random pseudomagnetic fields [36] that not only scatter charge carriers, but also break the effective time-reversal symmetry in a single valley [26,27] on approximately the same time scale. If these random pseudomagnetic fields are the dominant source of scattering limiting the mobility, we can immediately understand why $\tau$ and $\tau_{*}$ are comparable. On the contrary, for a potential $V$ generated by charged impurities on and in the substrate, $\tau$ is determined by the Fourier components $V(k)$ with $k \approx k_{F}$, whereas $\tau_{*}$ is determined by random fluctuations in the potential difference between the $A$ and $B$ atoms in the individual unit cells of graphene, i.e., by the Fourier component of $V$ with $k \simeq 1 / a$ (see Appendix C). Since $V$ is a long-range potential, $V\left(k_{F}\right) \gg$ $V(1 / a)$, implying (through Fermi's golden rule) that for charged impurities $\tau_{*} \gg \tau$, in disagreement with the experimental observations. We are not aware of any mechanism other than strain-induced pseudomagnetic fields that can explain the coincidence between $\tau$ and $\tau_{*}$, which is why the indication of this finding for the relevance of local strain fluctuations is rather compelling.

Albeit less directly, the experimentally observed evolution of $\mu$ upon annealing also points to the effect of strain. As discussed above, repeated annealing at low temperature $\left(\simeq 200^{\circ} \mathrm{C}\right)$ in an inert atmosphere systematically reduces $\mu$ by 1 order of magnitude. These annealing processes have no significant chemical effect and, therefore, are not expected to change the density of charge at the surface of hBN by 1 order of magnitude (as would be needed to explain the changes in $\mu$ [13]). On the contrary, they do lead to visible mechanical deformations, compatible with strain causing a decrease in mobility. Finally, having $\mu$ limited by strain-induced pseudomagnetic fields also explains why the use of high- $\epsilon$ substrates - such as $\mathrm{SrTiO}_{3}$ [19]—does not lead to a very large increase in mobility: a high- $\epsilon$ substrate can screen scalar potentials, but not the effect of a pseudomagnetic field.

\section{RAMAN MAPPING FOR CORRELATING STRAIN FLUCTUATIONS AND CARRIER MOBILITY}

Additional indications that carrier mobility in graphene is limited by local strain fluctuations can be obtained by combining transport measurements with spatially resolved Raman spectroscopy [37,38]. The quantity of interest in this case is the linewidth of the Raman $2 \mathrm{D}$ peak $\Gamma_{2 \mathrm{D}}$. In contrast to the width of the $G$ peak, $\Gamma_{2 \mathrm{D}}$ only very weakly depends on doping, charge inhomogeneities [39-41], or magnetic field [21]. $\Gamma_{2 \mathrm{D}}$ is also only weakly affected by global strain and by the different screening properties of the substrates $[42,43]$, while it is highly sensitive to strain inhomogeneities on length scales smaller the laserspot size $(<500 \mathrm{~nm})$, as recently shown by Neumann and co-workers [21]. These are precisely the random strain fluctuations that can contribute to scattering of charge carriers.

Figure 5(a) shows the inverse mobility $\mu^{-1}$ versus the linewidth of the $2 \mathrm{D}$ peak for a number of contacted 

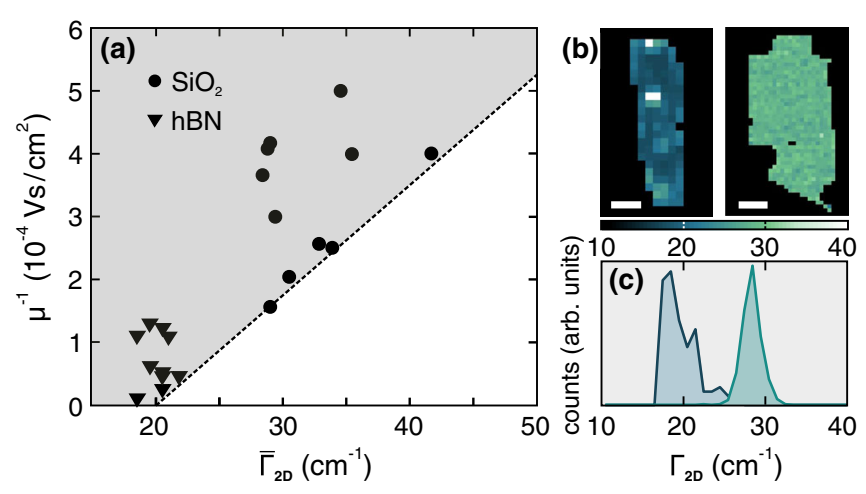

FIG. 5. (a) Correlation of the inverse mobility and the average full width at half maximum of the Raman $2 \mathrm{D}$ peak $\Gamma_{2 \mathrm{D}}$ for a number of graphene flakes on different substrates. (b) Raman maps of two graphene flakes resting on two different substrates (left, $\mathrm{hBN}$; right, $\mathrm{SiO}_{2}$ ) highlighting the different values of the spatially resolved $\Gamma_{2 \mathrm{D}}$ (same color scale). The white scale bars are $2 \mu \mathrm{m}$. (c) Histograms of $\Gamma_{2 D}$ for the two examples shown in (b). These histograms are used to extract the data points illustrated in (a).

graphene flakes resting on different substrates. Each of the data points corresponds to a different sample, on which we perform low-temperature $(T=1.8 \mathrm{~K})$ transport measurements to extract the mobility $\mu$, as well as spatially resolved Raman maps, such as those of Fig. 5(b) (the color code represents the linewidth $\Gamma_{2 \mathrm{D}}$ of the local $2 \mathrm{D}$ peak). From these maps we extract for each flake the distribution of $\Gamma_{2 \mathrm{D}}$, see Fig. 5(c), from which we calculate the average width $\bar{\Gamma}_{2 \mathrm{D}}$ [this is the quantity plotted on the horizontal axis of Fig. 5(a)]. Values of $\bar{\Gamma}_{2 \mathrm{D}}$ larger than the intrinsic linewidth of the 2D peak are indicative of strain fluctuations in the graphene layer [21], and a larger $\bar{\Gamma}_{2 \mathrm{D}}$ corresponds to a larger magnitude of these random strain fluctuations.

Finding that the data points from all the investigated devices in Fig. 5(a) lie above the dotted lines means that the maximum observed value of $\mu$ is smaller in devices for which $\bar{\Gamma}_{2 \mathrm{D}}$ is larger, i.e., in devices with larger random strain fluctuations. This directly indicates that strain is limiting the carrier mobility. The data show a rather large spread in mobility values, which originates from the fact that the mobility can be limited by structural defects-like folds formed in graphene during the transfer and fabrication process-which can have only a small effect on the averaged linewidth $\bar{\Gamma}_{2 \mathrm{D}}$. Indeed, the devices used for these combined Raman and transport measurements were not etched to confine transport through regions in which these types of structural defects are absent, since etching would have drastically reduced the area of graphene, making Raman measurements considerably more complex. As a result, a quasi-one-dimensional fold or ripple cutting across the graphene flake [see, e.g., white regions in Fig. 5(b), left panel] can have a very strong effect on the mobility value extracted in the device, while-as it affects only a small part of the total device area-it has only a small effect on the averaged linewidth $\bar{\Gamma}_{2 \mathrm{D}}$. Despite these experimental limitations, the absence of data points in the nonshaded area indicates that a necessary condition to observe high carrier mobility values is to have small random strain fluctuations, and the correlation between maximum mobility and averaged linewidth $\bar{\Gamma}_{2 \mathrm{D}}$ is clearly apparent in the data.

\section{QUANTITATIVE EXPLANATION OF THE $1 / \mu-n^{*}$ CORRELATION IN TERMS OF STRAIN}

Having found a direct correlation between the strength of the random local strain in graphene and the carrier mobility-and therefore having confirmed the role of strain fluctuations as an important source of disorder-we check, for consistency, whether the relation between $1 / \mu$ and $n^{*}$ that we discussed earlier [see Figs. 3(a) and 3(b)] can be explained theoretically in terms of strain fluctuations only. As we mentioned previously, such a relation has been reported experimentally earlier on, in the study of transport through graphene exposed to an increasingly large density of potassium atoms, where it was naturally explained in terms of the effect of charged impurities (the ionized potassium atoms) [11]. Below, we show that the relation between $1 / \mu$ and $n^{*}$ is very naturally reproduced also if random local strain is the dominant source of disorder. Indeed, at the quantitative level, the experimental data agree with theoretical calculations for realistic values (i.e., in the range known from literature) of the elastic parameters of graphene, which describe the coupling between strain and electronic properties.

Strain can originate from both in-plane and out-of-plane deformations (the latter being the so-called ripples), with the former being probably the most relevant ones, especially on hBN substrates. The effect of random strain fluctuations on the motion of electrons in graphene can be described by introducing a scalar and a vector potential $V_{s}$ and $\mathbf{A}$ in the long-wavelength Dirac Hamiltonian. What is needed to calculate the effect of strain fluctuations on $\mu$ and $n^{*}$ are the correlation functions of these potentials, which can all be obtained directly from the correlation function of the random strain field (as described in Appendix A). The scalar and the gauge potential scatter electrons (with rates $1 / \tau_{s}$ and $1 / \tau_{g}$, respectively) and limit the mobility $\mu$. The magnitude of the charge fluctuations $n^{*}$, on the contrary, is determined by the scalar potential only. We calculate $1 / \tau_{s}$ and $1 / \tau_{g}$ using Fermi's golden rule and obtain the total scattering time as $1 / \tau=1 / \tau_{s}+1 / \tau_{g}$ :

$$
\begin{aligned}
\frac{1}{\tau_{s}}= & \frac{2 \pi N\left(E_{F}\right)}{\hbar^{2}} \frac{\pi^{2}}{} \\
& \times\left.\int_{0}^{\pi} d \theta \frac{1-\cos ^{2}(\theta)}{2} \frac{\left\langle V_{s}(\mathbf{q}) V_{s}(-\mathbf{q})\right\rangle}{\epsilon^{2}(\mathbf{q})}\right|_{|\mathbf{q}|=2 k_{F} \sin (\theta / 2)}
\end{aligned}
$$

and 


$$
\begin{aligned}
\frac{1}{\tau_{g}}= & \frac{2 \pi}{\hbar^{2}} \frac{N\left(E_{F}\right)}{4 \pi^{2}} \\
& \times\left.\int_{0}^{\pi} d \theta[1-\cos (\theta)]\left\langle\mathbf{A}_{\perp}(\mathbf{q}) \mathbf{A}_{\perp}(-\mathbf{q})\right\rangle\right|_{|\mathbf{q}|=2 k_{F} \sin (\theta / 2)},
\end{aligned}
$$

where $\mathbf{A}_{\perp}(\mathbf{q})$ is the component of $\mathbf{A}$ perpendicular to $\mathbf{q}$, $N\left(E_{F}\right)=\left(k_{F} /\left(2 \pi \hbar v_{F}\right)\right)$ is the one-valley density of states at the Fermi energy, $\epsilon(\mathbf{q})=\left(\epsilon_{0}+1\right) / 2+4 e^{2} k_{F} / v_{F}|\mathbf{q}|$ is the dielectric function including the substrate contribution, and $k_{F}, v_{F}$, and $E_{F}$ are the Fermi momentum, velocity, and energy. We extract the mobility from $\mu=\sigma / n e=$ $2\left(e^{2} / h\right)\left(E_{F} \tau / \hbar n e\right)$ (the factor of 2 accounts for the two valleys). To calculate the magnitude of charge fluctuations, we use the relation $n(\mathbf{r})=(1 / \pi)\left(V_{s}(\mathbf{r}) / \hbar v_{F}\right)^{2}$ between local charge density and potential, from which

$$
n^{*}=\frac{1}{\pi} \frac{\left\langle V_{s}(\mathbf{r})^{2}\right\rangle}{\left(\hbar v_{F}\right)^{2}}=\frac{1}{4 \pi^{3} \hbar^{2} v_{F}^{2}} \int d^{2} \mathbf{q} \frac{\left\langle V_{s}(\mathbf{q}) V_{s}(-\mathbf{q})\right\rangle}{\epsilon^{2}(\mathbf{q})}
$$

Since the correlation functions of all the potentials are determined by the same correlation function describing the random strain field, $\mu$ and $n^{*}$ are related. We find in all cases a linear relation between $1 / \mu$ and $n^{*}$ (within logarithmic corrections) with a slope determined by the elastic coefficients of graphene, whose specific expression differs for out-of-plane and for in-plane strain. For out-of-plane strain, we have

$$
\frac{1}{\mu}=n^{*} \frac{h}{4 e}\left[\frac{\hbar^{2} v_{F}^{2}}{8 e^{4}}+\frac{g_{2}^{2}\left(\lambda_{L}+\mu_{L}\right)^{2}}{g_{1}^{2} \mu_{L}^{2}}\right] \frac{1}{\log \left[1 /\left(k_{F}\left(n^{*}\right) a\right)\right]},
$$

whereas for in-plane strain we obtain

$$
\begin{aligned}
\frac{1}{\mu}= & n^{*} \frac{h}{4 e}\left[\frac{\hbar^{2} v_{F}^{2}}{16 e^{4}}+\frac{g_{2}^{2}}{g_{1}^{2}}\left(1+\frac{\left(\lambda_{L}+2 \mu_{L}\right)^{2}}{\mu_{L}^{2}}\right)\right] \\
& \times \frac{1}{\log \left[1 /\left(k_{F}\left(n^{*}\right) a\right)\right]} .
\end{aligned}
$$

In both expressions, the first term in the square brackets originates from the contribution to scattering of the scalar potential and the second originates from the contribution of the pseudomagnetic field. In these expressions, $g_{1}$ and $g_{2}$ quantify the strength of electron-phonon coupling in graphene, $\mu_{L}=9.4 \mathrm{eV} / \AA^{2}$ and $\lambda_{L}=3.3 \mathrm{eV} / \AA^{2}$ are Lamé coefficients [44], and $\left(e^{2} / \hbar v_{F}\right)=2.2(a$ is the lattice constant of graphene and the logarithm appears when cutting off the integrals at large $q$ values, at $q=1 / a$ ). Equations (5) and (6) show that the relation between $1 / \mu$ and $n^{*}$ is linear (the deviations caused by the logarithm are within the fluctuations in the data, and in fact improve the overall agreement), as found experimentally. Notably, these relations depend on only fundamental constants and on the elastic properties of graphene. In this regard, the only role of the substrate is to determine the magnitude of the strain present in the graphene lattice.

The dashed lines in Figs. 3(a) and 3(b) are best fits to the data $\left((1 / \mu)=(h / e) n^{*} \times 0.118\right)$. Both expressions above for random out-of-plane or in-plane strain reproduce this value of the slope with realistic values of the $g_{1}$ and $g_{2}$ parameters (the slope depends on only their ratio). The parameter $g_{2}$ is determined by the modulation of the hopping between $p_{z}$ orbitals, and it can be extracted from measurements of effective magnetic fields created in highly strained graphene [45]. A reasonable value is $g_{2} \approx 2.5 \mathrm{eV}$ [46]. The parameter $g_{1}$ gives the strength of the scalar potential, and estimates of its magnitude vary in the range $g_{1} \approx 4-10 \mathrm{eV}$ [46-49]. Using $n^{*}=10^{11} \mathrm{~cm}^{-2}$ and fixing $g_{2}=2.5 \mathrm{eV}$, the expression for random strain due to ripples [Eq. (5)] reproduces the slope of the $1 / \mu-n^{*}$ relation for $g_{1}=3.65 \mathrm{eV}$, and if Eq. (6) for in-plane strain is taken, the experimental value is obtained for $g_{1}=6.9 \mathrm{eV}$, in all cases fully compatible with the expected range of values. We conclude that random strain quantitatively accounts for the $1 / \mu-n^{*}$ relation observed in the experiments. While both in-plane and out-of-plane random strain contribute, it is likely that on hBN substrates in-plane strain dominates.

Having fixed the values of $g_{2} / g_{1}$ by comparing the theoretical expression for $1 / \mu$ with the experimental data, we can determine whether it is the scalar or the gauge potential originating from strain that gives the dominant contribution to the scattering time. Interestingly, we find that for both out-of-plane and in-plane random strain, the scattering time associated with the random gauge potential $\tau_{g}$ is approximately 1 order of magnitude smaller than the scattering time associated with the scalar potential $\tau_{s}$; i.e., it is the gauge potential that poses the most stringent limit to the mobility. This is exactly what we would expect from our analysis of weak localization, and specifically from the experimental observation that $\tau_{*} \simeq \tau$. This finding also explains why the use of high dielectric constant substrates (such as $\mathrm{SrTiO}_{3}$ ) cannot lead to a major increase in mobility [19]: a high- $\epsilon$ substrate could screen the deformation potential-which is electrostatic in nature-but not the effect of a random pseudomagnetic field. We conclude that our theoretical analysis of the $1 / \mu-n^{*}$ relation does not only reproduce the experimental data with realistic values of the model parameters, but it is also internally consistent with other independent experimental observations. It is this level of quantitative agreement and internal consistency of results obtained by means of different techniques that strongly supports the validity of our interpretation.

\section{CONCLUSIONS}

The experimental and theoretical results discussed above lead to a consistent physical scenario that can be understood only if random strain fluctuations are the dominant source of disorder in graphene on $\mathrm{hBN}$ (and other substrates). We summarize the key points. The analysis of 
weak-localization measurements shows that $\tau_{i v} \gg \tau$, implying that scattering of charge carriers occurs mainly within the same valley, and that it is, therefore, due to a long-range potential. It also shows that the characteristic time to break the effective single-valley time-reversal symmetry $\tau_{*}$ is comparable to $\tau$, the elastic scattering time extracted from the mobility, a finding that can be explained naturally if random pseudomagnetic fields due to strain are the dominant scattering mechanism. Since this finding $\left(\tau_{*} \simeq \tau\right)$ does not appear to be compatible with any other disorder mechanism, the indication that it provides as to the relevance of random strain fluctuations is particularly compelling. The role of local strain fluctuations is further confirmed by the correlation between the maximum observed mobility and the line width of the Raman 2D peak measured on the very same devices (which has been identified as a measure of the intensity of local mechanical deformations, i.e., local strain). Finally, a conceptually straightforward theoretical analysis shows that strain provides a qualitative and quantitative understanding of the linear relation between $1 / \mu$ and $n^{*}$. This same analysis confirms that strain-induced disorder mainly generates scattering through random pseudomagnetic fields, and not through the scalar deformation potential, which is precisely what we had concluded independently through the study of weak localization.

Although most considerations above have been made for graphene on hBN, our results point to the relevance of strain fluctuation also for graphene on $\mathrm{SiO}_{2}$ and $\mathrm{SrTiO}_{3}$ substrates. Indeed, data obtained from devices on $\mathrm{SiO}_{2}$ and $\mathrm{SrTiO}_{3}$ satisfy quantitatively the same $1 / \mu-n^{*}$ relation that we have found analyzing many devices on hBN. For graphene on $\mathrm{SiO}_{2}$, weak-localization measurements done in the past $[28,29]$ allow us to draw conclusions similar to those that we have discussed here for devices on hBN. Additionally, random strain fluctuations explain why devices made on substrates with extremely different surface chemistry show similar mobility $(\approx 5.000-$ $10.000 \mathrm{~cm}^{2} / \mathrm{V} \mathrm{s}$ ), a fact that would be difficult to understand if charge impurities at the substrate surface were the dominating source of disorder (simply because the density of charged impurities should depend very strongly on the specific chemical groups present at the substrate surface). Finally, the finding that strain fluctuations dominantly couple to the electrons through the generation of a random pseudomagnetic field - and not through the deformation potential-explains why the mobility in devices on $\mathrm{SrTiO}_{3}$ substrates [19], which have a very high dielectric constant, is not much higher than on $\mathrm{SiO}_{2}$, since the effect of magnetic field cannot be screened electrostatically.

\section{ACKNOWLEDGMENTS}

A. F. M. gratefully acknowledges support by the Swiss National Science Foundation (SNF) and by the National Center of Competence in Research on Quantum Science and Technology (NCCR QSIT). F. G. acknowledges support from the Spanish Ministry of Economy (MINECO) through Grant No. FIS2011-23713 and the European Research Council (ERC) Advanced Grant (Contract No. 290846). C. S. and S. E. acknowledge experimental help from F. Buckstegge, J. Dauber, B. Terrés, F. Volmer, and M. Drögeler and financial support from Deutsche Forschungsgemeinschaft (DFG) and European Research Council (ERC) (Contract No. 280140). A. F. M., F. G., and C. S. acknowledge funding from the European Union (EU) under the Graphene Flagship.

\section{APPENDIX A: ANALYSIS OF STRAIN DISTRIBUTIONS}

We discuss the technical details of the analysis of the effects of random strain and derive the expressions for the relations between $1 / \mu$ and $n^{*}$ reported in the main text. Strain can be induced either by out-of-plane corrugations or by in-plane displacements of the atoms in the graphene lattice. We analyze the two cases separately. We emphasize that this same analysis is consistent with the observed density dependence of the mobility: irrespective of whether strain is in-plane or out-of-plane, the calculated mobility is independent of carrier density, within logarithmic corrections that cause a slow mobility suppression at large $n$.

\section{Out-of-plane corrugations}

We assume a given height profile $h(\overrightarrow{\mathbf{r}})$. The height corrugations lead to strain, which induce a scalar and a gauge potential acting on the electrons [36]:

$$
\begin{aligned}
& V_{s}(\overrightarrow{\mathbf{q}})=-g_{1} \frac{\mu_{L}}{\lambda_{L}+2 \mu_{L}} \frac{q_{x}^{2}+q_{y}^{2}}{|\overrightarrow{\mathbf{q}}|^{4}} \mathcal{F}(\overrightarrow{\mathbf{q}}), \\
& A_{x}(\overrightarrow{\mathbf{q}})=g_{2} \frac{\lambda_{L}+\mu_{L}}{\lambda_{L}+2 \mu_{L}} \frac{q_{x}^{2}-q_{y}^{2}}{|\overrightarrow{\mathbf{q}}|^{4}} \mathcal{F}(\overrightarrow{\mathbf{q}}), \\
& A_{y}(\overrightarrow{\mathbf{q}})=-2 g_{2} \frac{\lambda_{L}+\mu_{L}}{\lambda_{L}+2 \mu_{L}} \frac{q_{x} q_{y}}{|\overrightarrow{\mathbf{q}}|^{4}} \mathcal{F}(\overrightarrow{\mathbf{q}}),
\end{aligned}
$$

where $g_{1}$ and $g_{2}$ are parameters with dimensions of energy, $\lambda_{L}$ and $\mu_{L}$ are the elastic Lamé coefficients. $\mathcal{F}(\overrightarrow{\mathbf{q}})=$ $\sum_{i, j} q_{i} q_{j} f_{i, j}(\overrightarrow{\mathbf{q}})-|\overrightarrow{\mathbf{q}}|^{2} \sum_{i} f_{i, i}(\overrightarrow{\mathbf{q}})$, with $f_{i, j}(\overrightarrow{\mathbf{q}})$ the Fourier transform of $f_{i, j}(\overrightarrow{\mathbf{r}})=\partial_{i} h(\overrightarrow{\mathbf{r}}) \partial_{j} h(\overrightarrow{\mathbf{r}}), g_{2}=3 c \beta \gamma_{0} / 4$, with $\gamma_{0} \approx 2.7 \mathrm{eV}, \quad \beta=\partial \log \left(\gamma_{0}\right) / \partial \log (a) \approx 2$, and $c=\mu_{L} /$ $\left[\sqrt{2}\left(\lambda_{L}+\mu_{L}\right)\right] \approx 0.59[44]$.

We assume that the height correlations are such that

$$
\langle h(\overrightarrow{\mathbf{q}}) h(-\overrightarrow{\mathbf{q}})\rangle=\frac{A}{|\overrightarrow{\mathbf{q}}|^{4}},
$$

where $A$ is a constant. This dependence corresponds to the profile of a membrane with temperature $k_{B} T \propto A \kappa$, where $\kappa$ is the bending rigidity of graphene [35]. This assumption leads to $\langle\mathcal{F}(\overrightarrow{\mathbf{q}}) \mathcal{F}(-\overrightarrow{\mathbf{q}})\rangle=\bar{A}|\overrightarrow{\mathbf{q}}|^{2}$, where $\bar{A}$ is a dimensionless constant. It is given, approximately, by 


$$
\bar{A} \sim \frac{h_{r}^{4}}{\ell_{r}^{4}},
$$

where $h_{r}$ and $\ell_{r}$ are typical values for the height and size of the ripples. Using Eqs. (2) and (3), we find

$$
\begin{aligned}
& \tau_{s}^{-1} \approx \begin{cases}\frac{v_{F} g_{1}^{2} \mu_{L}^{2} \bar{A}}{32 \pi\left(\lambda_{L}+2 \mu_{L}\right)^{2} e^{4} k_{F}}+\cdots & \frac{\epsilon_{0} \hbar v_{F}}{e^{2}} \lesssim 1 \\
\frac{g_{1}^{2} \mu_{L}^{2} \bar{A}}{8 \pi\left(\lambda_{L}+2 \mu_{L}\right)^{2}\left(\epsilon_{0}+1\right)^{2} \hbar^{2} v_{F} k_{F}}+\cdots & \frac{\epsilon_{0} \hbar v_{F}}{e^{2}} \gtrsim 1,\end{cases} \\
& \tau_{g}^{-1} \approx \frac{g_{2}^{2}\left(\lambda_{L}+\mu_{L}\right)^{2} \bar{A}}{4 \pi \hbar^{2} v_{F} k_{F}\left(\lambda_{L}+2 \mu_{L}\right)^{2}} .
\end{aligned}
$$

The mobility is given by $\mu=\sigma /(n e)=2 \frac{e^{2}}{h} \frac{v_{F} k_{F} \tau}{n e}=\frac{2 e \pi}{h} \frac{v_{F} \tau}{k_{F}}$.

For $\epsilon_{0} \lesssim e^{2} /\left(\hbar v_{F}\right)$, the mobility is

$$
\frac{1}{\mu}=\frac{\hbar \bar{A}}{e}\left[\frac{g_{1}^{2} \mu_{L}^{2}}{32 \pi e^{4}\left(\lambda_{L}+2 \mu_{L}\right)^{2}}+\frac{g_{2}^{2}\left(\lambda_{L}+\mu_{L}\right)^{2}}{4 \pi\left(\hbar v_{F}\right)^{2}\left(\lambda_{L}+2 \mu_{L}\right)^{2}}\right] .
$$

The scalar potential in Eq. (A1) gives rise to charge fluctuations, whose amplitude is given by

$$
\begin{aligned}
n^{*} & =\frac{\left\langle V_{s}^{2}(\overrightarrow{\mathbf{r}})\right\rangle}{\pi \hbar^{2} v_{F}^{2}} \\
& =\frac{1}{4 \pi^{3} \hbar^{2} v_{F}^{2}} \int d^{2} \overrightarrow{\mathbf{q}} \frac{\left\langle V_{s}(\overrightarrow{\mathbf{q}}) V_{s}(-\overrightarrow{\mathbf{q}})\right\rangle}{\epsilon^{2}(\overrightarrow{\mathbf{q}})} \\
& \approx \frac{g_{1}^{2} \mu_{L}^{2} \bar{A}}{2 \pi^{2}\left(\lambda_{L}+2 \mu_{L}\right)^{2}\left(\hbar v_{F}\right)^{2}} \log \left(\frac{1}{k_{F}\left(n^{*}\right)}\right) .
\end{aligned}
$$

The ratio of the two expressions above leads to the $1 / \mu-n^{*}$ relation, Eq. (5) in the main text.

\section{In-plane strain}

A supporting substrate induces forces on the carbon atoms of a graphene layer, leading to strain and deformations. Therefore, in addition to deformations that can occur on a corrugated substrate, or because of imperfect adhesion during the graphene transfer process, in-plane forces on the carbon atoms can also be expected. These forces induce strain, which modify the electronic properties. In particular, periodic interactions, associated with the incommensuration between the lattices of graphene and the substrate, lead to the formation of superstructures and moiré patterns [50-52]. In addition, a random distribution of forces should be expected, due to impurities in the substrates and other imperfections in the graphene-substrate system (e.g., remnants of adsorbates in between the substrate and graphene).

We neglect the short-range, periodic component of the interaction potential between graphene and the substrate, and consider a random potential $V(\overrightarrow{\mathbf{r}})$, which varies slowly over a distance $\xi \gg a$, where $a$ is the lattice constant:

$$
\left\langle V(\overrightarrow{\mathbf{r}}) V\left(\overrightarrow{\mathbf{r}}^{\prime}\right)\right\rangle \approx \bar{V}^{2} \xi^{2} \delta\left(\overrightarrow{\mathbf{r}}-\overrightarrow{\mathbf{r}}^{\prime}\right) .
$$

This potential leads to forces at the positions of the carbon atoms:

$$
\overrightarrow{\mathbf{F}}(\overrightarrow{\mathbf{r}})=\nabla V(\overrightarrow{\mathbf{r}}) .
$$

The elastic energy of the graphene lattice is

$$
\begin{aligned}
\mathcal{H}_{\text {elastic }}= & \frac{\lambda}{2} \int d^{2} \overrightarrow{\mathbf{r}}\left(\sum_{i=x, y} u_{i i}\right)^{2}+\mu \int d^{2} \overrightarrow{\mathbf{r}} \sum_{i, j=x, y} u_{i j}^{2} \\
& +\int \frac{d^{2} \overrightarrow{\mathbf{r}}}{A} \overrightarrow{\mathbf{F}}(\overrightarrow{\mathbf{r}}) \vec{u}(\overrightarrow{\mathbf{r}}),
\end{aligned}
$$

where $A=\sqrt{3} d_{G}^{2} / 2$ is the area of the unit cell, $d_{G}$ is the lattice constant, $u(\overrightarrow{\mathbf{r}})$ is the displacement of the atom at position $\overrightarrow{\mathbf{r}}$ from its equilibrium position, $u_{i j}=$ $\left(\partial_{i} u_{j}+\partial_{j} u_{i}\right) / 2$, and we assume that the displacements are small, so that the assumption of a linear coupling to local forces is valid.

We Fourier transform Eq. (A9):

$$
\begin{aligned}
\mathcal{H}_{\text {elastic }}= & \frac{\lambda}{2} \sum_{\overrightarrow{\mathbf{k}}}\left(\overrightarrow{\mathbf{k}}_{\overrightarrow{\mathbf{k}}}\right)^{2}+\mu \sum_{\overrightarrow{\mathbf{k}}} \frac{\left(k_{i} u_{j}+k_{j} u_{i}\right)^{2}}{4} \\
& +\sum_{\overrightarrow{\mathbf{k}}} \frac{\overrightarrow{\mathbf{F}}_{\overrightarrow{\mathbf{k}}} \overrightarrow{\mathbf{u}}_{\overrightarrow{\mathbf{k}}}}{A} .
\end{aligned}
$$

For long wavelength force distributions, $|\overrightarrow{\mathbf{k}}| \ll|\overrightarrow{\mathbf{G}}|$, where $\overrightarrow{\mathbf{G}}$ is a reciprocal vector of the graphene lattice, the displacements are

$$
\overrightarrow{\mathbf{u}}_{\overrightarrow{\mathbf{k}}}=-\frac{\overrightarrow{\mathbf{F}}_{\overrightarrow{\mathbf{k}}}^{\|}}{A(\lambda+2 \mu)|\overrightarrow{\mathbf{k}}|^{2}}-\frac{\overrightarrow{\mathbf{F}}_{\overrightarrow{\mathbf{k}}}^{\perp}}{A \mu|\overrightarrow{\mathbf{k}}|^{2}},
$$

where the $\|$ and $\perp$ superscripts stand for the parallel and transverse components of $\overrightarrow{\mathbf{F}}_{\overrightarrow{\mathbf{k}}}$ with respect to $\overrightarrow{\mathbf{k}}$ $\left(\overrightarrow{\mathbf{F}}_{\overrightarrow{\mathbf{k}}}=i \overrightarrow{\mathbf{k}} V_{\overrightarrow{\mathbf{k}}}\right.$, the vector $\overrightarrow{\mathbf{F}}_{\overrightarrow{\mathbf{k}}}$ has only a longitudinal component, but this is not the generic case; see below). If $|\overrightarrow{\mathbf{k}}-\overrightarrow{\mathbf{G}}|=|\delta \overrightarrow{\mathbf{k}}| \ll|\overrightarrow{\mathbf{G}}|$, long wavelength displacements are also generated,

$$
\overrightarrow{\mathbf{u}}_{\delta \overrightarrow{\mathbf{k}}}=-\frac{\overrightarrow{\mathbf{F}}_{\overrightarrow{\mathbf{k}}}^{\|}}{A(\lambda+2 \mu)|\delta \overrightarrow{\mathbf{k}}|^{2}}-\frac{\overrightarrow{\mathbf{F}}_{\overrightarrow{\mathbf{k}}}^{\perp}}{A \mu|\delta \overrightarrow{\mathbf{k}}|^{2}},
$$

where now the superscripts $\|$ and $\perp$ refer to the orientation of $\overrightarrow{\mathbf{F}}_{\overrightarrow{\mathbf{k}}}$ with respect to $\delta \overrightarrow{\mathbf{k}}$. From $\overrightarrow{\mathbf{u}}_{\overrightarrow{\mathbf{k}}}$, we can obtain the strain tensor

$$
u_{i, j}(\overrightarrow{\mathbf{k}})=\frac{\left.k_{i} \overrightarrow{\mathbf{u}}_{\overrightarrow{\mathbf{k}}}\right|_{j}+\left.k_{j} \overrightarrow{\mathbf{u}}_{\overrightarrow{\mathbf{k}}}\right|_{i}}{2} .
$$

For $|\overrightarrow{\mathbf{k}}| \ll|\overrightarrow{\mathbf{G}}|$, we have $\quad \overrightarrow{\mathbf{F}}_{\overrightarrow{\mathbf{k}}}=\overrightarrow{\mathbf{k}} V_{\overrightarrow{\mathbf{k}}}$, while for $|\delta \mathbf{k}|=|\overrightarrow{\mathbf{k}}-\overrightarrow{\mathbf{G}}| \ll|\overrightarrow{\mathbf{G}}|$, we obtain $\overrightarrow{\mathbf{F}}_{\delta \overrightarrow{\mathbf{k}}} \approx \overrightarrow{\mathbf{G}} V_{\overrightarrow{\mathbf{G}}}$. Thus, we 
obtain two different contributions to the correlations of long-range strain:

$$
\begin{aligned}
\left\langle u_{i, j}(\overrightarrow{\mathbf{k}}) u_{i, j}(-\overrightarrow{\mathbf{k}})\right\rangle & \propto\langle V(\overrightarrow{\mathbf{k}}) V(-\overrightarrow{\mathbf{k}})\rangle, \\
\left\langle u_{i, j}(\delta \overrightarrow{\mathbf{k}}) u_{i, j}(-\delta \overrightarrow{\mathbf{k}})\right\rangle & \propto \frac{|\overrightarrow{\mathbf{G}}|^{2}}{|\overrightarrow{\mathbf{k}}|^{2}}\langle V(\overrightarrow{\mathbf{G}}) V(-\overrightarrow{\mathbf{G}})\rangle,
\end{aligned}
$$

where $\langle V(\overrightarrow{\mathbf{k}}) V(-\overrightarrow{\mathbf{k}})\rangle=\bar{V}^{2} \xi^{2}$. In the following, we concentrate on the effects of the random components of the potential of order $|\overrightarrow{\mathbf{G}}|=(4 \pi) /\left(\sqrt{3} d_{G}\right)$. The contribution to the transport properties is larger that from small momenta by terms of order $|\overrightarrow{\mathbf{G}}|^{2} / k_{F}^{2}$.

The scalar and gauge potentials are

$$
\begin{aligned}
V_{s}(\delta \overrightarrow{\mathbf{k}})= & g_{1} \sum_{\overrightarrow{\mathbf{G}}} \frac{\left(G_{x} \delta k_{x}+G_{y} \delta k_{y}\right) V_{\overrightarrow{\mathbf{k}}}}{\left(\lambda_{L}+2 \mu_{L}\right)|\delta \overrightarrow{\mathbf{k}}|^{2}}, \\
A_{x}(\delta \overrightarrow{\mathbf{k}})= & \frac{g_{2}}{\hbar v_{F}} \sum_{\overrightarrow{\mathbf{G}}} \frac{V_{\overrightarrow{\mathbf{k}}}}{|\delta \mathbf{k}|^{4}} \\
& \times\left\{\left[\left(\delta k_{x}\right)^{2}-\left(\delta k_{y}\right)^{2}\right] \frac{G_{x} \delta k_{x}+G_{y} \delta k_{y}}{\lambda_{L}+2 \mu_{L}}\right. \\
& \left.+\left(2 \delta k_{x} \delta k_{y}\right) \frac{-G_{x} \delta k_{y}+G_{y} \delta k_{x}}{\mu_{L}}\right\}, \\
A_{y}(\delta \overrightarrow{\mathbf{k}})= & \frac{g_{2}}{\hbar v_{F}} \sum_{\overrightarrow{\mathbf{G}}} \frac{V_{\overrightarrow{\mathbf{k}}}}{|\delta \mathbf{k}|^{4}} \\
& \times\left\{-\left(2 \delta k_{x} \delta k_{y}\right) \frac{G_{x} \delta k_{x}+G_{y} \delta k_{y}}{\lambda_{L}+2 \mu_{L}}\right. \\
& \left.+\left[\left(\delta k_{x}\right)^{2}-\left(\delta k_{y}\right)^{2}\right] \frac{-G_{x} \delta k_{y}+G_{y} \delta k_{x}}{\mu_{L}}\right\} .
\end{aligned}
$$

The gauge potential can be divided into a component $\overrightarrow{\mathbf{A}}_{\|}$, parallel to $\delta \overrightarrow{\mathbf{k}}$, and a component $\overrightarrow{\mathbf{A}}_{\perp}$, perpendicular to $\delta \overrightarrow{\mathbf{k}}$. The effect of $\overrightarrow{\mathbf{A}}_{\|}$can be gauged away, and only $\overrightarrow{\mathbf{A}}_{\perp}$ gives a physical effect. In terms of the potential correlations, we find

$$
\begin{aligned}
& \left\langle V_{s}(\delta \overrightarrow{\mathbf{k}}) V_{s}(-\delta \overrightarrow{\mathbf{k}})\right\rangle \\
& =\sum_{\overrightarrow{\mathbf{G}}} \frac{g_{1}^{2}|\overrightarrow{\mathbf{G}}|^{2} \bar{V}^{2} \xi^{2} \cos ^{2}(\theta)}{\left(\lambda_{L}+2 \mu_{L}\right)^{2}|\delta \overrightarrow{\mathbf{k}}|^{2} A^{2}}, \\
& \left\langle\overrightarrow{\mathbf{A}}_{\perp}(\delta \overrightarrow{\mathbf{k}}) \overrightarrow{\mathbf{A}}_{\perp}(-\delta \overrightarrow{\mathbf{k}})\right\rangle \\
& =\sum_{\overrightarrow{\mathbf{G}}} \frac{g_{2}^{2}|\overrightarrow{\mathbf{G}}|^{2} \bar{V}^{2} \xi^{2}}{\left(\hbar v_{F}\right)^{2}|\delta \overrightarrow{\mathbf{k}}|^{2} A^{2}} \\
& \times\left[\frac{\sin ^{2}(3 \theta) \cos ^{2}(\theta)}{\left(\lambda_{L}+2 \mu_{L}\right)^{2}}+\frac{\cos ^{2}(3 \theta) \sin ^{2}(\theta)}{\mu_{L}^{2}}\right],
\end{aligned}
$$

where $\theta$ is the angle between $\overrightarrow{\mathbf{G}}$ and $\delta \overrightarrow{\mathbf{k}}, A=d_{G}^{2} \sqrt{3} / 2$ is the area of the unit cell of the graphene lattice, and we neglect terms proportional to quantities like $\sin (\theta) \cos (\theta)$, which average to zero when summing over $\overrightarrow{\mathbf{G}}$. The factor $\cos (3 \theta)$ arises from extracting the component of $\overrightarrow{\mathbf{A}}$ normal to $\delta \overrightarrow{\mathbf{k}}$. Using Eqs. (2) and (3), we obtain

$$
\begin{aligned}
& \frac{\hbar}{\tau_{s}} \approx \frac{\pi g_{1}^{2} \bar{V}^{2} \xi^{2}}{6\left(\lambda_{L}+2 \mu_{L}\right)^{2} d_{G}^{6} \alpha^{2} \hbar v_{F} k_{F}}, \\
& \frac{\hbar}{\tau_{g}} \approx \frac{8 \pi g_{2}^{2} \bar{V}^{2} \xi^{2}}{3 d_{G}^{6} \hbar v_{F} k_{F}}\left[\frac{1}{\left(\lambda_{L}+2 \mu_{L}\right)^{2}}+\frac{1}{\mu_{L}^{2}}\right] .
\end{aligned}
$$

The mobility is

$$
\frac{1}{\mu}=\frac{e n}{\sigma}=\frac{h}{e} \frac{k_{F}}{2 \pi v_{F}}\left(\frac{1}{\tau_{s}}+\frac{1}{\tau_{g}}\right) .
$$

The carrier fluctuations at the neutrality point are given by

$$
n^{*} \approx \frac{16 g_{1}^{2} \bar{V}^{2} \xi^{2}}{3\left(\hbar v_{F}\right)^{2}\left(\lambda_{L}+2 \mu_{L}\right)^{2} d_{G}^{6}} \log \left(\frac{1}{k_{F}\left(n^{*}\right) a}\right) .
$$

From the ratio of the last two expressions, we obtain Eq. (6) in the main text.

\section{APPENDIX B: $1 / \mu-n^{*}$ RELATION FOR BILAYER GRAPHENE}

Here, we illustrate that the linearity of the relation between $\mu^{-1}$ and $n^{*}$ for monolayer graphene is not trivial, by comparing the result shown in Fig. 3 to those of a similar analysis done for bilayer graphene devices (the bilayer devices have been fabricated on $\mathrm{hBN}$ and $\mathrm{SiO}_{2}$ substrates, following protocols identical to those used for the

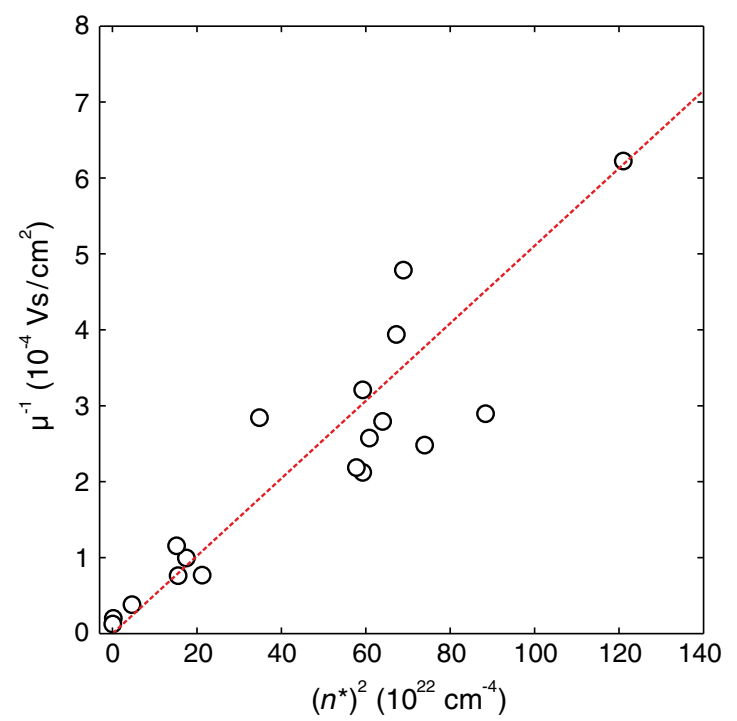

FIG. 6. Correlation of the inverse mobility and $\left(n^{*}\right)^{2}$ for a number of different bilayer graphene flakes on different substrates. 
monolayers). The result is illustrated in Fig. 6, in which we plot $\mu^{-1}$ as a function of $\left(n^{*}\right)^{2}$ : it is apparent that the experimental data obey a linear relation, i.e., for bilayers $\mu^{-1} \propto\left(n^{*}\right)^{2}$, in contrast to the relation $\mu^{-1} \propto\left(n^{*}\right)$ found in monolayers. This finding further supports our theoretical analysis of disorder in monolayers in terms of strain, which correctly captures the observed, nontrivial linear dependence of the relation between $1 / \mu$ and $n^{*}$.

\section{APPENDIX C: WEAK LOCALIZATION AND SINGLE-VALLEY EFFECTIVE TIME-REVERSAL SYMMETRY}

Weak-localization measurements in graphene provide a wealth of information about the scattering processes that take place in the material. As we discuss in the main text, we can conclude directly from the results of the fits of the magnetoresistance curves that the intervalley scattering time $\tau_{i v}$ is much longer than the elastic scattering time $\tau$, which indicates that intravalley scattering processesand therefore long-range potentials - dominate the effect of disorder. We also find that the elastic scattering time $\tau$ nearly coincides with the time $\tau_{*}$ needed to break the effective single-valley time-reversal symmetry, and argue that this observation strongly indicates that random strainand not charged impurities-is the dominant scattering source. As the reader may not be fully familiar with the concept of effective single-valley time-reversal symmetry, we discuss this here in some more detail for completeness (for more information, see Ref. [27]).

Effective single-valley time-reversal symmetry is a concept relevant for graphene, in the regime in which a continuum Dirac Hamiltonian provides a good description (i.e., when the Fermi level is not too far away from the charge neutrality point). In the ideal case, the Dirac Hamiltonian (where $\mathbf{k}$ is the momentum relative to the $K$ point)

$$
H=\hbar v_{F}\left(\begin{array}{cc}
0 & k_{x}+i k_{y} \\
k_{x}-i k_{y} & 0
\end{array}\right)
$$

is invariant upon the antiunitary transformation $i \hat{\sigma_{y}} \hat{K}$, with $\hat{K}$ denoting complex conjugation. This antiunitary transformation mimics the implementation of time-reversal symmetry, as - for each electronic state-it sends $\mathbf{k}$ into $-\mathbf{k}$ and reverses the spin. However, this is not the true timereversal symmetry operation. Indeed, true time-reversal symmetry changes the sign of the total momentum (and not just of the momentum relative to the $K$ point) and sends states in one of the valleys into the other valley (timereversal symmetry therefore cannot be implemented by considering only one valley). That is why $i \hat{\sigma}_{y} \hat{K}$ is referred to as "effective single-valley time-reversal symmetry." While it remains a good symmetry as long as $H$ is well approximated by the Dirac Hamiltonian, the implication of not being the "true" time-reversal operation is that it can be easily violated by different microscopic mechanisms.

For instance, effective time-reversal symmetry is violated by the quadratic momentum terms that are neglected when making the linear approximation in the continuum, which leads to the Dirac Hamiltonian. It is obvious thatbeing quadratic-these terms do not change sign upon inverting the sign of $\mathbf{k}$, whereas the linear terms in the Dirac Hamiltonian do. As a result, when including both the linear and quadratic terms in $\mathbf{k}$, the single-valley Hamiltonian is not invariant upon effective single-valley time-reversal symmetry. This mechanism, however, cannot account for our experimental observations $\left(\tau_{*} \simeq \tau\right.$ independent of carrier density): the effect of the quadratic terms becomes more relevant as $E_{F}$ is increased further away from the charge neutrality point. If these terms were the relevant ones in determining the characteristic time scale $\tau_{*}$, we should find that $\tau_{*}$ becomes shorter at larger carrier density, contrary to what we observe experimentally [see Fig. 4(c)]. Additionally, this mechanism cannot explain why $\tau_{*} \simeq \tau$, because the quadratic terms in $\mathbf{k}$ do not cause any scattering of electron waves.

The other two mechanisms that break effective timereversal symmetry are strain and the presence of a "gap" term in the Dirac Hamiltonian. Strain breaks the effective time-reversal symmetry by generating a random pseudomagnetic field. Indeed, within a single valley, this pseudomagnetic field acts on the orbital degrees of freedom in all regards as a true magnetic field, i.e., it is described by a gauge potential minimally coupled to the momentum. If the dominant source of scattering is spatial inhomogeneities in this gauge potential, such a mechanism very naturally explains why the elastic scattering time $\tau$ and the time needed to break the effective time-reversal symmetry $\tau_{*}$ nearly coincide, as scattering and effective time-reversal symmetry breaking originate from the same term in the Hamiltonian.

A gap term-i.e., a difference $\Delta$ in on-site energy between the $A$ and $B$ carbon atom in the unit cell of graphene-also breaks the effective single-valley timereversal symmetry. In that case, the Hamiltonian becomes

$$
H=\left(\begin{array}{cc}
\Delta / 2 & \hbar v_{F}\left(k_{x}+i k_{y}\right) \\
\hbar v_{F}\left(k_{x}-i k_{y}\right) & -\Delta / 2
\end{array}\right) .
$$

The fact that this Hamiltonian is not invariant upon $i \hat{\sigma}_{y} \hat{K}$ can be checked by a direct calculation. It is also obvious without doing any calculation, if we observe that this Hamiltonian is formally identical to that of Rashba spin-orbit interaction in the presence of a Zeeman term (with $g \mu B$ corresponding to $\Delta / 2$ ), a system that lacks timereversal symmetry.

In our experimental case, charged impurities on a substrate can contribute to the breaking of effective time-reversal symmetry through this mechanism. More 
specifically, charge impurities would generate random electrostatic potentials. On average, these potentials would be the same on the $A$ and $B$ atoms forming graphene. Nevertheless, fluctuations would exist so that locally the electrostatic on-site energy would be slightly different on the $A$ and $B$ atom in each unit cell, i.e., locally a nonzero $\Delta$ term would be present. However, in our experiments, this mechanism cannot explain why the scattering time $\tau$ and the characteristic time for breaking effective time-reversal symmetry $\tau_{*}$ would coincide. In fact, as discussed in the main text, this mechanism would predict that $\tau_{*} \gg \tau$, because $\tau$ is determined by the Fourier components of the potential at $k_{F}$, whereas $\Delta$ is determined by the components at $k \simeq 1 / a$ (with a long-ranged potential, the latter are much smaller).

[1] C. R. Dean, A. F. Young, I. Meric, C. Lee, L. Wang, S. Sorgenfrei, K. Watanabe, T. Taniguchi, P. Kim, K. L. Shepard, and J. Hone, Boron Nitride Substrates for HighQuality Graphene Electronics, Nat. Nanotechnol. 5, 722 (2010).

[2] P. J. Zomer, S. P. Dash, N. Tombros, and B. J. van Wees, A Transfer Technique for High Mobility Graphene Devices on Commercially Available Hexagonal Boron Nitride, Appl. Phys. Lett. 99, 232104 (2011).

[3] A. S. Mayorov, R. V. Gorbachev, S. V. Morozov, L. Britnell, R. Jalil, L. A. Ponomarenko, P. Blake, K. S. Novoselov, K. Watanabe, T. Taniguchi, and A. K. Geim, MicrometerScale Ballistic Transport in Encapsulated Graphene at Room Temperature, Nano Lett. 11, 2396 (2011).

[4] L. Wang, I. Meric, P. Y. Huang, Q. Gao, Y. Gao, H. Tran, T. Taniguchi, K. Watanabe, L. M. Campos, D. A. Muller, J. Guo, P. Kim, J. Hone, K. L. Shepard, and C. R. Dean, One-Dimensional Electrical Contact to a Two-Dimensional Material, Science 342, 614 (2013).

[5] C. R. Dean, A. F. Young, P. Cadden-Zimansky, L. Wang, H. Ren, K. Watanabe, T. Taniguchi, P. Kim, J. Hone, and K. L. Shepard, Multicomponent Fractional Quantum Hall Effect in Graphene, Nat. Phys. 7, 693 (2011).

[6] A. F. Young, C. R. Dean, L. Wang, H. Ren, P. CaddenZimansky, K. Watanabe, T. Taniguchi, J. Hone, K. L. Shepard, and P. Kim, Spin and Valley Quantum Hall Ferromagnetism in Graphene, Nat. Phys. 8, 550 (2012).

[7] L. A. Ponomarenko, R. V. Gorbachev, G. L. Yu, D. C. Elias, R. Jalil, A. A. Patel, A. Mishchenko, A. S. Mayorov, C. R. Woods, J. R. Wallbank, M. Mucha-Kruczynski, B. A. Piot, M. Potemski, I. V. Grigorieva, K. S. Novoselov, F. Guinea, V. I. Fal'ko, and A. K. Geim, Cloning of Dirac Fermions in Graphene Superlattices, Nature (London) 497, 594 (2013).

[8] C. R. Dean, L. Wang, P. Maher, C. Forsythe, F. Ghahari, Y. Gao, J. Katoch, M. Ishigami, P. Moon, M. Koshino, T. Taniguchi, K. Watanabe, K. L. Shepard, J. Hone, and P. Kim, Hofstadter's Butterfly and the Fractal Quantum Hall Effect in Moiré Superlattices, Nature (London) 497, 598 (2013).

[9] B. Hunt, J. D. Sanchez-Yamagishi, A. F. Young, M. Yankowitz, B. J. LeRoy, K. Watanabe, T. Taniguchi,
P. Moon, M. Koshino, P. Jarillo-Herrero, and R. C. Ashoori, Massive Dirac Fermions and Hofstadter Butterfly in a van der Waals Heterostructure, Science 340, 1427 (2013).

[10] A. H. Castro Neto, F. Guinea, N. M. R. Peres, K. S. Novoselov, and A. K. Geim, The Electronic Properties of Graphene, Rev. Mod. Phys. 81, 109 (2009).

[11] J. H. Chen, C. Jang, S. Adam, M. S. Fuhrer, E. D. Williams, and M. Ishigami, Charged-Impurity Scattering in Graphene, Nat. Phys. 4, 377 (2008).

[12] J.-H. Chen, W. G. Cullen, C. Jang, M. S. Fuhrer, and E. D. Williams, Defect Scattering in Graphene, Phys. Rev. Lett. 102, 236805 (2009).

[13] S. Adam, E. H. Hwang, V. M. Galitski, and S. Das Sarma, A Self-Consistent Theory for Graphene Transport, Proc. Natl. Acad. Sci. U.S.A. 104, 18392 (2007).

[14] C. Jang, S. Adam, J.-H. Chen, E. D. Williams, S. Das Sarma, and M.S. Fuhrer, Tuning the Effective Fine Structure Constant in Graphene: Opposing Effects of Dielectric Screening on Short- and Long-Range Potential Scattering, Phys. Rev. Lett. 101, 146805 (2008).

[15] L. A. Ponomarenko, R. Yang, T. M. Mohiuddin, M. I. Katsnelson, K. S. Novoselov, S. V. Morozov, A. A. Zhukov, F. Schedin, E. W. Hill, and A. K. Geim, Effect of a High-א Environment on Charge Carrier Mobility in Graphene, Phys. Rev. Lett. 102, 206603 (2009).

[16] N. M. R. Peres, Colloquium: The Transport Properties of Graphene: An Introduction, Rev. Mod. Phys. 82, 2673 (2010).

[17] M. Monteverde, C. Ojeda-Aristizabal, R. Weil, K. Bennaceur, M. Ferrier, S. Guéron, C. Glattli, H. Bouchiat, J. N. Fuchs, and D. L. Maslov, Transport and Elastic Scattering Times as Probes of the Nature of Impurity Scattering in Single-Layer and Bilayer Graphene, Phys. Rev. Lett. 104, 126801 (2010).

[18] S. Das Sarma, S. Adam, E. H. Hwang, and E. Rossi, Electronic Transport in Two-Dimensional Graphene, Rev. Mod. Phys. 83, 407 (2011).

[19] N. J. G. Couto, B. Sacepe, and A. F. Morpurgo, Transport through Graphene on $\mathrm{SrTiO}_{3}$, Phys. Rev. Lett. 107, 225501 (2011).

[20] S. Adam, S. Jung, N. N. Klimov, N. B. Zhitenev, J. A. Stroscio, and M. D. Stiles, Mechanism for Puddle Formation in Graphene, Phys. Rev. B 84, 235421 (2011).

[21] C. Neumann, S. Reichardt, M. Drogeler, K. Watanabe, T. Taniguchi, B. Beschoten, S. V. Rotkin, and C. Stampfer, Magneto-Raman Microscopy for Probing Local Material Properties of Graphene, arXiv:1406.7771.

[22] T. Taychatanapat, K. Watanabe, T. Taniguchi, and P. JarilloHerrero, Electrically Tunable Transverse Magnetic Focusing in Graphene, Nat. Phys. 9, 225 (2013).

[23] J.-H. Chen, C. Jang, S. Xiao, M. Ishigami, and M. S. Fuhrer, Intrinsic and Extrinsic Performance Limits of Graphene Devices on $\mathrm{SiO}_{2}$, Nat. Nanotechnol. 3, 206 (2008).

[24] J. Martin, N. Akerman, G. Ulbricht, T. Lohmann, J. H. Smet, K. von Klitzing, and A. Yacoby, Observation of Electron-Hole Puddles in Graphene Using a Scanning Single-Electron Transistor, Nat. Phys. 4, 144 (2008).

[25] In Ref. [19], we discussed transport through graphene on $\mathrm{SrTiO}_{3}$ in the context of resonant scattering, but we also pointed out-in Ref. [21] of that Letter-that the data are compatible with scattering by ripples, i.e., with the conclusions of this present work. 
[26] E. McCann, K. Kechedzhi, V. I. Fal'ko, H. Suzuura, T. Ando, and B. L. Altshuler, Weak-Localization Magnetoresistance and Valley Symmetry in Graphene, Phys. Rev. Lett. 97, 146805 (2006).

[27] A. F. Morpurgo and F. Guinea, Intervalley Scattering, LongRange Disorder, and Effective Time-Reversal Symmetry Breaking in Graphene, Phys. Rev. Lett. 97, 196804 (2006).

[28] F. V. Tikhonenko, D. W. Horsell, R. V. Gorbachev, and A. K. Savchenko, Weak Localization in Graphene Flakes, Phys. Rev. Lett. 100, 056802 (2008).

[29] J. Guignard, D. Leprat, D. C. Glattli, F. Schopfer, and W. Poirier, Quantum Hall Effect in Exfoliated Graphene Affected by Charged Impurities: Metrological Measurements, Phys. Rev. B 85, 165420 (2012).

[30] D. K. Ferry and S. M. Goodnick, Transport in Nanostructures (Cambridge University Press, Cambridge, England, 2009).

[31] A. M. R. Baker, J. A. Alexander-Webber, T. Altebaeumer, T. J. B. M. Janssen, A. Tzalenchuk, S. Lara-Avila, S. Kubatkin, R. Yakimova, C.-T. Lin, L.-J. Li, and R. J. Nicholas, Weak Localization Scattering Lengths in Epitaxial, and CVD Graphene, Phys. Rev. B 86, 235441 (2012).

[32] S. Engels, B. Terrés, A. Epping, T. Khodkov, K. Watanabe, T. Taniguchi, B. Beschoten, and C. Stampfer, Limitations to Carrier Mobility and Phase-Coherent Transport in Bilayer Graphene, Phys. Rev. Lett. 113, 126801 (2014).

[33] T. Ando, Screening Effect and Impurity Scattering in Monolayer Graphene, J. Phys. Soc. Jpn. 75, 074716 (2006).

[34] K. Nomura and A. H. MacDonald, Quantum Transport of Massless Dirac Fermions, Phys. Rev. Lett. 98, 076602 (2007).

[35] M. I. Katsnelson and A. K. Geim, Electron Scattering on Microscopic Corrugations in Graphene, Phil. Trans. R. Soc. A 366, 195 (2008).

[36] M. A. H. Vozmediano, M. I. Katsnelson, and F. Guinea, Gauge Fields in Graphene, Phys. Rep. 496, 109 (2010).

[37] D. Graf, F. Molitor, K. Ensslin, C. Stampfer, A. Jungen, C. Hierold, and L. Wirtz, Spatially Resolved Raman Spectroscopy of Single- and Few-Layer Graphene, Nano Lett. 7, 238 (2007).

[38] J. E. Lee, G. Ahn, J. Shim, Y. S. Lee, and S. Ryu, Optical Separation of Mechanical Strain from Charge Doping in Graphene, Nat. Commun. 3, 1024 (2012).

[39] S. Pisana, M. Lazzeri, C. Casiraghi, K. S. Novoselov, A. K. Geim, A.C. Ferrari, and F. Mauri, Breakdown of the
Adiabatic Born-Oppenheimer Approximation in Graphene, Nat. Mater. 6, 198 (2007).

[40] C. Stampfer, F. Molitor, D. Graf, K. Ensslin, A. Jungen, C. Hierold, and L. Wirtz, Raman Imaging of Doping Domains in Graphene on $\mathrm{SiO}_{2}$, Appl. Phys. Lett. 91, 241907 (2007).

[41] S. Berciaud, X. Li, H. Htoon, L. E. Brus, S. K. Doorn, and T. F. Heinz, Intrinsic Line Shape of the Raman 2D-Mode in Freestanding Graphene Monolayers, Nano Lett. 13, 3517 (2013).

[42] V. N. Popov and P. Lambin, Theoretical 2D Raman Band of Strained Graphene, Phys. Rev. B 87, 155425 (2013).

[43] F. Forster, A. Molina-Sanchez, S. Engels, A. Epping, K. Watanabe, T. Taniguchi, L. Wirtz, and C. Stampfer, Dielectric Screening of the Kohn Anomaly of Graphene on Hexagonal Boron Nitride, Phys. Rev. B 88, 085419 (2013).

[44] K. V. Zakharchenko, M. I. Katsnelson, and A. Fasolino, Finite Temperature Lattice Properties of Graphene beyond the Quasiharmonic Approximation, Phys. Rev. Lett. 102, 046808 (2009).

[45] N. Levy, S. A. Burke, K. L. Meaker, M. Panlasigui, A. Zettl, F. Guinea, A. H. Castro Neto, and M. F. Crommie, StrainInduced Pseudo-magnetic Fields Greater than 300 Tesla in Graphene Nanobubbles, Science 329, 544 (2010).

[46] M. Gibertini, A. Tomadin, F. Guinea, M. I. Katsnelson, and M. Polini, Electron-Hole Puddles in the Absence of Charged Impurities, Phys. Rev. B 85, 201405 (2012).

[47] S. Ono and K. Sugihara, Theory of the Transport Properties in Graphite, J. Phys. Soc. Jpn. 21, 861 (1966).

[48] H. Suzuura and T. Ando, Phonons and Electron-Phonon Scattering in Carbon Nanotubes, Phys. Rev. B 65, 235412 (2002).

[49] S.-M. Choi, S.-H. Jhi, and Y.-W. Son, Effects of Strain on Electronic Properties of Graphene, Phys. Rev. B 81, 081407 (2010).

[50] C. R. Woods et al., Commensurate-Incommensurate Transition in Graphene on Hexagonal Boron Nitride, Nat. Phys. 10, 451 (2014).

[51] J. Jung, A. DaSilva, S. Adam, and A. H. MacDonald, Origin of Band Gaps in Graphene on Hexagonal Boron Nitride, arXiv:1403.0496.

[52] P. San-Jose, A. Gutiérrez-Rubio, M. Sturla, and F. Guinea, Spontaneous Strains and Gap in Graphene on Boron Nitride, Phys. Rev. B 90, 075428 (2014). 